\title{
The effects of antifungal stewardship programs at a tertiary-care teaching hospital in Japan
}

Hiroshi Kawaguchi, Koichi Yamada, Waki Imoto, Kazushi Yamairi, Wataru Shibata, Hiroki Namikawa, Naoka Yoshii, Kiyotaka Nakaie, Yasuyo Okada, Akiko Fujita, Yoshikatsu Shinoda, Yasutaka Nakamura, Yukihiro Kaneko, Katsuya Nagayama, Hiroshi Kakeya

\begin{tabular}{|c|l|}
\hline Citation & Journal of Infection and Chemotherapy, 25(6); 458-462 \\
\hline Issue Date & $2019-06$ \\
\hline Type & Journal Article \\
\hline Textversion & author \\
\hline Rights & $\begin{array}{l}\text { O2019 Japanese Society of Chemotherapy and The Japanese Association for Infectious } \\
\text { Diseases. This manuscript version is made available under the CC-BY-NC-ND 4.0 License. } \\
\text { https://creativecommons.org/licenses/by-nc-nd/4.0/ }\end{array}$ \\
$\begin{array}{l}\text { This is the accepted manuscript version. Please cite only the published version. The article } \\
\text { has been published in final form at https://doi.org/10.1016/j.jiac.2018.10.016 }\end{array}$ \\
\hline DOI & https://doi.org/10.1016/j.jiac.2019.01.015 \\
\hline
\end{tabular}

\author{
Self-Archiving by Author(s) \\ Placed on: Osaka City University
}

Kawaguchi, H., Yamada, K., Imoto, W., Yamairi, K., Shibata, W., Namikawa, H., Yoshii, N., Nakaie, K., Okada, Y., Fujita, A., Shinoda, Y., Nakamura, Y., Kaneko, Y., Nagayama, K., \& Kakeya, H. (2019). The effects of antifungal stewardship programs at a tertiary-care teaching hospital in Japan. Journal of Infection and Chemotherapy. 25, 458-462. https://doi.org/10.1016/j.jiac.2019.01.015 
The Effects of Antifungal Stewardship Programs at a Tertiary-Care Teaching Hospital in Japan

Hiroshi Kawaguchi ${ }^{\mathrm{a}, \mathrm{b}}$, Koichi Yamada ${ }^{\mathrm{b}, \mathrm{c}, \mathrm{d}}$, Waki Imoto ${ }^{\mathrm{b}}$, Kazushi Yamairi ${ }^{\mathrm{b}}$, Wataru Shibata $^{\mathrm{b}}$, Hiroki Namikawa ${ }^{\mathrm{b}}$, Naoka Yoshii ${ }^{\mathrm{b}}$, Kiyotaka Nakaie $^{\mathrm{b}}$, Yasuyo Okada ${ }^{\mathrm{d}}$, Akiko Fujita $^{\mathrm{d}}$, Yoshikatsu Shinoda ${ }^{\mathrm{a}}$, Yasutaka Nakamura ${ }^{\mathrm{a}}$, Yukihiro Kaneko ${ }^{\mathrm{c}}$, Katsuya Nagayama $^{\mathrm{a}}$ and Hiroshi Kakeya ${ }^{\mathrm{b}, \mathrm{c}, \mathrm{d}}$

a Department of Pharmacy, Osaka City University Hospital, 1-5-7, Asahi-machi, Abeno-ku, Osaka-shi, Osaka, 545-8586 Japan

${ }^{b}$ Department of Infection Control Science, Graduate School of Medicine, Osaka City University, 1-4-3, Asahi-machi, Abeno-ku, Osaka-shi, Osaka, 545-8585 Japan

${ }^{c}$ Research Center for Infectious Diseases, Graduate School of Medicine, Osaka City

University, 1-4-3, Asahi-machi, Abeno-ku, Osaka-shi, Osaka, 545-8585 Japan

${ }^{\mathrm{d}}$ Department of Infection Control and Prevention, Osaka City University Hospital, 1-5-7, Asahi-machi, Abeno-ku, Osaka-shi, Osaka, 545-8586 Japan

Corresponding Author: Hiroshi Kakeya, 1-4-3, Asahi-machi, Abeno-ku, Osaka-shi, Osaka, 545-8585 Japan

Phone: +81-6-6645-3784 
Facsimile: +81-6-6646-6056

E-mail: kakeya@med.osaka-cu.ac.jp

Authorship statement: All authors meet the ICMJE authorship criteria. 


\section{Abstract}

Patients with invasive fungal diseases (IFDs) generally have a high mortality rate, and resistance to antifungal drugs and the high costs associated with it have led to recent problems, necessitating the appropriate use of antifungals. To this end, we launched Antifungal Stewardship Programs (AFSPs) in our hospital. Patients who were systemically administered antifungals from January 2011 to December 2016 were enrolled this study and divided into pre-intervention and intervention groups. No significant difference was observed in defined daily doses per 1000 patient-days $(23.3 \pm 8.0$ vs $20.4 \pm 10.8, p=0.251)$ between the groups. The monthly average for the days of therapy per 1000 patient-days was significantly lower in the intervention group $(15.1 \pm 3.1$ vs $12.7 \pm 4.3, \mathrm{p}=0.009)$. The cost of the antifungals reduced over the 3 -year period by $\$ 260,520(13.5 \%)$. Furthermore, a decreasing trend was observed in both the 30-day mortality (40.9\% vs 30.0\%, p=0.414) and in-hospital mortality $(63.6 \%$ vs $36.7 \%$, $\mathrm{p}=0.054$ ) in patients with candidemia. Our results indicate that AFSPs are efficacious and cost-effective approaches.

Keywords: Antifungal stewardship programs (AFSPs), Defined daily doses per 1000 patient-days, Days of therapy per 1000 patient-days, Candidemia 


\section{Introduction}

In recent years, with advances in medical treatment there has also been an increase in immunocompromised patients, which has led to an increase in the incidence of invasive fungal diseases (IFDs). The diagnosis of IFDs is based on the presence of fungi in the blood, other sterile body sites, and deep tissue. IFDs are associated with a high mortality and are a major problem worldwide [1]. For the treatment of IFDs, azoles, echinocandins and liposomal amphotericin B (L-AMB) are used depending on the severity of the disease and the fungal species, azoles, echinocandins and liposomal amphotericin B (L-AMB) are used. There have been new developments in the identification of antifungals recently, and it is not expected that any new groundbreaking antifungals will be developed anytime soon. Furthermore, antifungals are costly, and their increased use would have a significant economic impact. For these reasons, antifungals must be used appropriately, with an understanding of their activity, pharmacokinetics, and safety. The antifungal stewardship programs (AFSPs) are designed to optimize the use of antifungal agents by integrating specialist experience and knowledge [2]. While the effectiveness of AFSPs have been reported $[3,4,5]$, there is limited research on them. Moreover, while there have been studies on the effectiveness of AFSPs in Europe and US $[3,4,5]$, there are few 
reports from Japan [6]. Here, we launched and put into practice AFSPs at our hospital, and we report their effectiveness in this study.

\section{Material and methods}

Antifungal stewardship programs

From January 2014, an Antimicrobial Stewardship Team (AST), including infectious disease physicians, pharmacists, medical biologists, and nurses was set up in the Osaka City University Hospital. The AST carried out activities that pertained to antimicrobial stewardship as well as those relevant to AFSPs. The activities that pertained to AFSPs included i) interventions for patients with blood cultures positive for yeasts, ii) the introduction of a conditional notification system for antifungals, and iii) the commencement of AST rounds. The candidemia management bundles that are recommended in Japan were followed for patients with yeast-positive blood cultures [7]. The AFSPs that we implemented are shown in Table 1.

\section{Study design and target group}

This was a single-center, observational study. The AFSPs commenced in January 2014 at the Osaka City University Hospital (980 beds, tertiary-care teaching hospital). We enrolled patients who received systemic antifungals from January 2011 to December 
2016. Patients treated between January 2011 and December 2013 were put into the pre-intervention group, and patients treated between January 2014 and December 2016 were put into the intervention group. The antifungals investigated in this study were L-AMB, caspofungin (CPFG), micafungin (MCFG), fosfluconazole (F-FLCZ), and voriconazole (VRCZ). CPFG was introduced from April 2012. The primary endpoints were the usage and the economic efficiency of the antifungals, and the secondary endpoint was mortality of patients with candidemia. Approval for this study was obtained from the Osaka City University ethics committee (approval number 3726). In accordance with "ethical guidelines for medical and health research involving human subjects", information on the implementation of study was published, individual written consent was not obtained from patients.

\section{Data collection}

All data were retrospectively examined. With regards to antifungal usage, the monthly defined daily doses (DDDs) per 1000 patient-days and days of therapy (DOTs) per 1000 patient-days were assessed. The DDDs/1000 patient-days were calculated in accordance with the World Health Organization (WHO) guidelines. The DOTs/1000 patient-days was calculated by multiplying the number of antifungal therapy days divided by the number of patients who were hospitalized by 1000 . The cost of antifungals was examined 
over three years after the commencement of the AFSPs based on the prices of antifungals in Japan in April 2018 and was then converted to US dollars (1 yen = US \$0.009). We examined the detected Candida species from all cultures during the study period. We also examined the details of the support provided to patients prescribed antifungals and the acceptance rate of the support after AFSPs implementation. Thirty-day mortality, in-hospital mortality, and the achievement rate of the bundles were examined in patients with candidemia.

\section{Statistical analysis}

The statistical analysis was performed using IBM SPSS® Statistics 22.0 (IBM Japan, Tokyo). The nominal variables of the two groups were compared using the Chi-square test or the Fisher's exact test, and the continuous variables were compared using the Student's t-test. Differences were considered statistically significant at a p-value of $<0.05$.

\section{Results}

\section{Patient characteristics}

Throughout the study period, there were 1793 patients who received antifungals. The patient characteristics of both groups are shown in Table 2. The usage frequency of 
VRCZ $(10.9 \%$ vs $5.8 \%, \mathrm{p}<0.001)$ and F-FLCZ $(12.6 \%$ vs $6.9 \%$, p<0.001) was significantly lower in the intervention group. Although the usage frequency of MCFG was significantly lower in the intervention group than in the pre-intervention group (50.9\% vs $43.7 \%, \mathrm{p}=0.002)$, the usage frequency of CPFG was significantly higher $(11.2 \%$ vs $32.4 \%, \mathrm{p}<0.001)$.

Antifungal usage and cost

The comparison of antifungal usage and cost between the groups is shown in Table 3 . The monthly average DDDs/1000 patient-days for all of the antifungals was not significantly different between the groups $(23.3 \pm 8.0$ vs $20.4 \pm 10.8, \mathrm{p}=0.251)$. The monthly average DOTs/1000 patient-days was significantly decrease in the intervention group $(15.1 \pm 3.1$ vs12.7 $\pm 4.3, \mathrm{p}=0.009)$. The cost of the antifungals was US $\$ 1,930,067$ for the pre-intervention group and US\$1,669,547 for the intervention group, demonstrating an antifungal cost reduction of US\$260,520 (13.5\%) over the 3-year period.

Candida species distribution during 2011-2016

All Candida species isolated during 2011-2016 are shown in Table 4. A total of 6,146 Candida isolates were identified from all cultures. There were no significant differences in the detections of the five major Candida species (Candida albicans, Candida 
glabrata, Candida parapsilosis, Candida tropicalis, and Candida krusei) between the pre-intervention and intervention. The other species detected included Candida rugosa, Candida guilliermondii, Candida lusitaniae and unidentified Candida isolates. These other species showed a significantly higher prevalence in the intervention period $(7.1 \%$ vs $8.5 \%, \mathrm{p}=0.04)$.

AFSPs support and acceptance rate

After the AFSPs had commenced, there were 214 antifungal prescription episodes for which support was provided. The support was provided for 131 episodes related to the selection or modification of antifungals (No. of acceptance episodes, and acceptance rate, 129/131, 98.5\%), 40 related to the discontinuation of antifungals (39/40, 97.5\%), 22 related to dosage modification $(21 / 22,95.5 \%)$, and 21 related to changes to oral treatment $(21 / 21,100 \%$,$) . The total acceptance rate of the support provided by the AFSPs was$ $98.1 \%$.

Candidemia prognosis and achievement rate of the bundles

The characteristics and prognostic results of patients with candidemia are shown in Table 5. No significant difference was observed between the groups with regards to patient characteristics. The 30-day mortality was $40.9 \%$ for the pre-intervention group 
and $30.0 \%$ for the post-intervention group ( $\mathrm{p}=0.414)$. The in-hospital mortality was $63.6 \%$ for the pre-intervention group and $36.7 \%$ for the post-intervention group $(p=0.054)$. The achievement rates of the bundles are shown in Table 6 . The number of ophthalmological examinations and repeat blood cultures after the start of therapy was significantly higher in the intervention group $(22.7 \%$ vs $70.0 \%, \mathrm{p}<0.001,50.0 \%$ vs $73.3 \%$, $\mathrm{p}=0.040$ ). The achievement of the key 7 elements (remove CV catheter within $24 \mathrm{~h}$ of diagnosis, appropriate selection of antifungals, appropriate dose of antifungals, ophthalmological examinations, repeat blood culture after the start of therapy, assessment of clinical effectiveness on the third to fifth day, 14 days of therapy after blood culture is confirmed to be negative) was significantly higher in the intervention group (13.6\% vs $50.0 \%, \mathrm{p}=0.006)$.

\section{Discussion}

In this study, AFSPs were initiated at our hospital, and the effectiveness of such programs was investigated. When implementing AFSPs, it is recommended that they are carried out by a multi-disciplinary team, which should at least include clinicians, pharmacists, and microbiologists [2]. Our hospital's AST is composed of infectious disease physicians, pharmacists, clinical laboratory technicians, and nurses, all of whom 
are members capable of implementing the AFSPs. In the AFSPs that we implemented, as soon as the blood culture tested positive for yeast, the infectious disease physicians confirmed whether or not the patient should undergo antifungal treatment. If the appropriate treatment had not been commenced, the infectious disease physicians recommended early treatment of candidemia.

Recently, due to problems such as low susceptibility and resistance of the candida species to the azoles, the use of echinocandins has been strongly recommended by the American and European guidelines [8,9] for candidemia. Reports in Japan have also found an increased detection of Candida glabrata, which has low susceptibility to the azoles [10]. As there were many patients from the department of hematology, department of surgery, and the emergency department in this study, many patients were likely suspected of having severe or invasive candidiasis. The frequency of azoles usage in the post-intervention group was lower, and the frequency of echinocandins usage was higher, likely owing to the appropriate selection of antifungals in the post-intervention group. In addition, because there was no significant increase in Candida glabrata detection during study period, the increase in echinocandin usage might be due to the increase in the number of cases that were at a stage higher than middle grade. Of the echinocandins, the frequency of MCFG usage decreased; however, this was due to the increase in CPFG usage after its introduction in 2012. 
Some previous studies have found DDDs to decrease when AFSPs are implemented [4], and such trends were similarly confirmed in this study. Prior to the commencement of the AFSPs, there were instances where insufficient doses of antifungals had been prescribed due to the fear of side effects. However, after the AFSPs commenced, the adequate antifungal dosage was selected because of the confirmation and support provided by infectious disease physicians. This increase in the antifungal dose likely explains why the DDDs/1000 patient-days did not decrease significantly. As interventions related to the judgment of the necessity of antifungals, long-term prescription checks, and the step-down of oral antifungals were carried out through the AFSPs, the DOTs/1000 patient-days significantly decreased. Hurst et al. found that after implementing ASPs, the antifungal DOTs decreased by $26.8 \%(\mathrm{p}<0.01)$ [11]. Similar results were obtained in this study. Over the 3-year period, the cost of antifungals was reduced by $\$ 260,520(13.5 \%)$. The decrease in antifungal usage and the decrease in the number of days for which the patient received the medication reflect the decrease in the cost of antifungals. In addition, the recommendation to discontinue unnecessary antifungals may have also contributed to the antifungal cost reduction. The antifungal cost reduction noted in our study was similar to that observed in previous studies $[4,12]$, which suggests that the AFSPs implemented in our institution are economically effective. 
Regarding the mortality of patients with candidemia, a decreasing trend was observed.

Takesue et al. found that compliance with the bundles for candidemia was revealed to be an independent predictor of mortality [7]. In this study, the significant increase in the achievement rate of the key 7 elements of the bundles for candidemia may be associated with the decrease in mortality of patients with candidemia. Reboli et al. found that more patients with candidemia died in the FLCZ group than in the anidulafungin group $(31.4 \%$ vs $22.8 \%, p=0.13)$ [13]. In this study, the increased usage of echinocandins may have been associated with a decrease in the mortality of patients with candidemia. Ramos et al. found that for patients with prescribed antifungals, the mortality rate was decreased from $30 \%$ to $17 \%$ ( $\mathrm{p}=0.393$ ) after AFSPs implementation [3]. Valerio M et al. found that the 30 -day mortality of candidemia decreased from $28.0 \%$ to $16.4 \%(\mathrm{p}=0.100)$ after AFSPs implementation [4]; and in agreement with the results of this study, there was a decreasing trend in the mortality as a result of the AFSPs implementation, although this decrease was not significant. The mortality in candidemia is high [14], and the seriousness of the underlying disease could explain this lack of significant decrease.

This study had a few limitations. First, as this was an AFSP initiative that was performed by a single institution. Second, the antifungals that can be used abroad and in Japan differ. Specifically, anidulafungin and posaconazole are used overseas, but are not available in Japan. As these differences in available antifungals may affect antifungal 
usage and cost, a comparative investigation of Japanese data that includes a uniform application of antifungals is necessary in the future. Third, the effect of AFSPs on patients with filamentous fungi could not be investigated in this study, because mycoses with filamentous fungi are difficult to diagnose and the number of cases was small.

In conclusion, the results of the AFSPs that were implemented at a tertiary teaching hospital in Japan demonstrate that as the appropriate selection of antifungals increases, a decrease in antifungal usage and cost reduction also occur, and there is a trend towards improved prognoses of patients with candidemia. These findings suggest that the AFSPs that we implemented were effective. Further studies about the effectiveness of AFSPs in Japan is needed.

\section{Acknowledgments}

We would like to thank Editage (www.editage.jp) for English language editing.

\section{Funding Source}

This study was supported by the Research Program on Emerging and Re-emerging

Infectious Diseases from the Japan Agency Development, AMED (Grant number JP

17fk0108208 and 18fk0108052h0002) and JSPS KAKENHI (Grant number 16K09939).

\section{Conflict of Interest}


Hiroshi Kakeya has received grant support from Pfizer Japan Inc., MSD KK, Astellas

Pharma Inc., and Dainippon Sumitomo Pharma Co., Ltd. 


\section{References}

[1] Webb BJ, Ferraro JP, Rea S, Kaufusi S, Goodman BE, Spalding J. Epidemiology and clinical features of invasive fungal infection in a US health care network. Open Forum Infect Dis $2018 ; 5: 1-8$

[2] Agrawal S, Barnes R, Brüggemann RJ, Rautemaa-Richardson R, Warris A. The role of the multidisciplinary team in antifungal stewardship. J Antimicrob Chemother 2016;71(Suppl 2):37-42.

[3] Ramos A, Pérez-Velilla C, Asensio A, Ruiz-Antorán B, Folguera C, Cantero M, et al. Antifungal stewardship in a tertiary hospital. Rev Iberoam Micol 2015;32:209-13.

[4] Valerio M, Muñoz P, Rodríguez CG, Caliz B, Padilla B, Gijón P. Antifungal stewardship in a tertiary-care institution : A bedside intervention. Clin Microbiol Infect 2015;21:492.e1-9.

[5] Mondain V, Lieutier F, Hasseine L, Gari-Toussaint M, Poiree M, Lions C, et al. A 6-year antifungal stewardship programme in a teaching hospital. Infection 2013;41:621-8.

[6] Morii D, Ichinose N, Yokozawa T, Oda T. Impact of an infectious disease specialist on antifungal use: an interrupted time-series analysis in a tertiary hospital in Tokyo. J Hosp Infect 2018;99:133-8.

[7] Takesue Y, Ueda T, Mikamo H, Oda S, Takakura S, Kitagawa Y, et al. Management bundles for candidaemia: the impact of compliance on clinical outcomes. J Antimicrob Chemother 
2015;70:587-93.

[8] Pappas PG, Kauffman CA, Andes DR, Clancy CJ, Marr KA, Ostrosky-Zeichner L, et al. Clinical practice guideline for the management of candidiasis: 2016 update by the infectious diseases society of America. Clin Infect Dis 2016;62:1-50.

[9] Cornely OA, Bassetti M, Calandra T, Garbino J, Kullberg BJ, Lortholary O, et al. ESCMID* guideline for the diagnosis and management of candida diseases 2012: non-neutropenic adult patients. Clin Microbiol Infect 2012;18(Suppl 7):19-37.

[10]Kakeya H, Yamada K, Kaneko Y, Yanagihara K, Tateda K, Maesaki S, et al. National trends in the distribution of candida species causing candidemia in Japan from 2003 to 2014: A report by the epidemiological investigation committee for human mycoses in Japan epidemiological investigation committee for human mycoses in Japan. Med Mycol J 2018;59:19-22.

[11] Hurst AL, Child J, Pearce K, Palmer C, Todd JK, Parker SK. Handshake stewardship: A highly effective rounding-based antimicrobial optimization service. Pediatr Infect Dis J 2016;35:1104-10.

[12] Micallef C, Aliyu SH, Santos R, Brown NM, Rosembert D, Enoch DA. Introduction of an antifungal stewardship programme targeting high-cost antifungals at a tertiary hospital in Cambridge, England. J Antimicrob Chemoter 2015;70:1908-11.

[13] Reboli AC, Rotstein C, Pappas PG, Chapman SW, Kett DH, Kumar D, et al. Anidulafungin versus fluconazole for invasive candidiasis. N Eng J Med 2007;24:2472-82. 
[14] Pappas PG, Rex JH, Lee J, Hamill RJ, Larsen RA, Powderly W, et al. A prospective observational study of candidemia: epidemiology, therapy, and influences on mortality in hospitalized adult and pediatric patients. Clin Infect Dis 2003;37:634-43. 


\section{Tables}

2 Table 1 Antifungal stewardship programs

\begin{tabular}{ll} 
Object of intervention & Actions \\
\hline 1. Confirm blood culture results for patients every \\
morning
\end{tabular}

2. Confirm appropriate empiric therapy for patients

Interventions for patients with blood cultures

with blood cultures positive for yeasts

positive for yeasts

3. Intervene if treatment is not being administered or if

there is no improvement in patients with blood cultures

positive for yeasts

4. Confirm new antifungal prescriptions on the next

business day

5. Intervene if patients with new antifungal

Conditional notification system for antifungals

prescriptions do not receive appropriate antifungals,

review the prescription dose and blood cultures

6. Recommendations for monitoring the therapeutic

level of voriconazole for patients prescribed this drug 
patients with blood cultures positive and those who

have received antifungal prescriptions for longer than

14 days

8. Recommendations for the discontinuation of

unnecessary antifungal

9. Recommendations for removing the CV catheter

within $24 \mathrm{~h}$ of diagnosis

10. Recommendations for the appropriate selection

Bundles at the start of therapy for candidemia

and dose of antifungals

11. Recommendations for the ophthalmological

examinations

12. Recommendation for repeat blood cultures after

the start of therapy

13. Assessment of clinical efficacy on the third to fifth

Bundles after the initiation of therapy for

day

candidemia

14. Recommendations of 14 days of therapy after

blood culture is confirmed to be negative

15. Recommendation to step-down to oral treatment 
3 Table 2 Characteristics of patients enrolled in this study and the antifungals prescribed

\begin{tabular}{|c|c|c|c|}
\hline & Pre-intervention & Intervention group & p-value \\
\hline & group $(n=978)$ & $(\mathrm{n}=815)$ & \\
\hline Sex (male/female) & $563 / 415$ & $507 / 308$ & $0.046^{\text {a) }}$ \\
\hline Age (years) & $47.2 \pm 23.1$ & $47.8 \pm 22.9$ & $0.601^{b)}$ \\
\hline Under 18 years $(\%)$ & $145(14.8)$ & $131(16.1)$ & $0.466^{\text {a) }}$ \\
\hline \multicolumn{4}{|l|}{ Department (\%) } \\
\hline Hematology & $429(43.9)$ & $389(47.7)$ & $0.102^{\text {a) }}$ \\
\hline Pediatric & $144(14.7)$ & $126(15.5)$ & $0.664^{\text {a) }}$ \\
\hline Urology & $61(6.2)$ & $32(3.9)$ & $0.028^{\text {a) }}$ \\
\hline Gastrointestinal & $55(5.6)$ & $60(7.4)$ & $0.135^{\text {a) }}$ \\
\hline \multicolumn{4}{|l|}{ surgery } \\
\hline Emergency & $54(5.5)$ & $44(5.4)$ & $0.909^{\text {a) }}$ \\
\hline Others & $235(24.0)$ & $164(20.1)$ & $0.048^{\text {a) }}$ \\
\hline \multicolumn{4}{|l|}{ Antifungals (\%) } \\
\hline L-AMB & $140(14.3)$ & $92(11.3)$ & $0.057^{\text {a) }}$ \\
\hline VRCZ & $107(10.9)$ & $47(5.8)$ & $<0.001^{\text {a) }}$ \\
\hline F-FLCZ & $123(12.6)$ & $56(6.9)$ & $<0.001^{\text {a) }}$ \\
\hline
\end{tabular}


MCFG

$498(50.9)$

$356(43.7)$

$0.002^{\text {a) }}$

CPFG

$110(11.2)$

$264(32.4)$

$<0.001^{\text {a) }}$

4 Data are presented as mean \pm standard deviation, or No. of patients (\%)

$5 \quad{ }^{\text {a) }}$ Chi-square test, ${ }^{\text {b) }}$ Student's t-test

6 L-AMB: liposomal amphotericin B, VRCZ: voriconazole, F-FLCZ: fosfluconazole,

7 MCFG: micafungin, CPFG: caspofungin

8 
9 Table 3 Antifungal DDDs/1000 patient-days, DOTs/1000 patient-days, and cost

\begin{tabular}{llll}
\hline & Pre-intervention & Intervention group & p-value \\
& group $(\mathrm{n}=978)$ & $(\mathrm{n}=815)$ & \\
\hline DDDs/1000 & $23.3 \pm 8.0$ & $20.4 \pm 10.8$ & $0.251^{*}$ \\
patient-days & & & \\
DOTs/1000 & $15.1 \pm 3.1$ & $12.7 \pm 4.3$ & $0.009^{*}$ \\
patient-days & & & \\
Cost of antifungals & $1,930,067$ & $1,669,547$ & \\
(US\$) & & & \\
\hline
\end{tabular}

10 Data are presented as mean \pm standard deviation

11 *Student's t-test

12 DDDs: defined daily doses, DOTs: days of therapy 
15 Table 4 Candida species distributions during 2011-2016

\begin{tabular}{llll}
\hline Candida species & Pre-intervention & Intervention period & $\mathrm{p}$ value \\
& period (2011-2013) & $(2014-2016)$ & \\
& $\mathrm{n}(\%)$ & $\mathrm{n}(\%)$ & \\
\hline Candida albicans & $1845(60.1)$ & $1841(59.8)$ & $0.819^{*}$ \\
Candida glabrata & $710(23.1)$ & $709(23.0)$ & $0.931^{*}$ \\
Candida & $36(1.2)$ & $47(1.5)$ & $0.229^{*}$ \\
parapsilosis & & & \\
Candida tropicalis & $200(6.5)$ & $166(5.4)$ & $0.063^{*}$ \\
Candida krusei & $59(1.9)$ & $51(1.7)$ & $0.433^{*}$ \\
Others & $219(7.1)$ & $263(8.5)$ & $0.040^{*}$
\end{tabular}

$16 *$ Chi-square test 
19 Table 5 Characteristics, causes of Candida, and mortality in patients with candidemia

\begin{tabular}{|c|c|c|c|}
\hline & Pre-intervention & Intervention group & $\mathrm{p}$-value \\
\hline & group $(n=22)$ & $(n=30)$ & \\
\hline Sex (male/female) & $17 / 5$ & $18 / 12$ & $0.190^{\text {a) }}$ \\
\hline Age (years) & $60.0 \pm 15.9$ & $59.0 \pm 21.3$ & $0.865^{b)}$ \\
\hline Under 18 years & $0(0)$ & $2(6.7)$ & $1.000^{a)}$ \\
\hline \multicolumn{4}{|l|}{ Department } \\
\hline Gastrointestinal & $4(18.2)$ & $8(26.7)$ & $0.473^{a)}$ \\
\hline \multicolumn{4}{|l|}{ surgery } \\
\hline Emergency & $5(22.7)$ & $3(10.0)$ & $0.260^{c)}$ \\
\hline Gastroenterology & $3(13.6)$ & $5(16.7)$ & $1.000^{\mathrm{c})}$ \\
\hline Hematology & $3(13.6)$ & $4(13.3)$ & $1.000^{c)}$ \\
\hline Others & $7(31.8)$ & $10(33.3)$ & $0.908^{a)}$ \\
\hline \multicolumn{4}{|l|}{ Candida groups } \\
\hline Candida albicans & $9(40.9)$ & $13(43.3)$ & $0.861^{\text {a) }}$ \\
\hline Candida glabrata & $6(27.3)$ & $5(16.7)$ & $0.495^{\mathrm{c})}$ \\
\hline Candida & $2(9.1)$ & $7(23.3)$ & $0.272^{\mathrm{c})}$ \\
\hline
\end{tabular}




$\begin{array}{llll}\text { Candida tropicalis } & 2(9.1) & 4(13.3) & 1.000^{\mathrm{c})} \\ \text { Candida krusei } & 3(13.6) & 1(3.3) & 0.299^{\mathrm{c})} \\ \text { Others } & 0(0) & 2(6.7) & 1.000^{\mathrm{c})} \\ \text { Mortality } & & & \\ \text { 30-day mortality } & 9(40.9) & 9(30.0) & 0.414^{\mathrm{a})} \\ \text { In-hospital } & 14(63.6) & 11(36.7) & 0.054^{\text {a) }} \\ \text { mortality } & & \end{array}$

20 Data are presented as mean \pm standard deviation, or $\mathrm{n}(\%)$

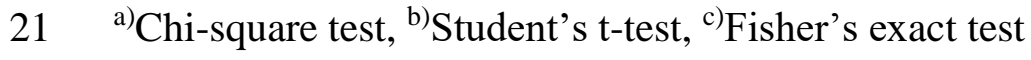


24 Table 6 Achievement rate of bundles for candidemia

\begin{tabular}{|c|c|c|c|}
\hline & $\begin{array}{l}\text { Pre-intervention } \\
\text { group }(n=22)\end{array}$ & $\begin{array}{l}\text { Intervention group } \\
(\mathrm{n}=30)\end{array}$ & p-value \\
\hline Remove CV & $11 / 17(64.7)$ & $17 / 22(77.3)$ & $0.390 * * *$ \\
\hline \multicolumn{4}{|l|}{ catheter within $24 \mathrm{~h}$} \\
\hline \multicolumn{4}{|l|}{ of diagnosis ${ }^{*}$} \\
\hline Appropriate & $18 / 22(81.8)$ & $27 / 30(90.0)$ & $0.390 * * *$ \\
\hline \multicolumn{4}{|l|}{ selection of } \\
\hline \multicolumn{4}{|l|}{ antifungals } \\
\hline Appropriate dose & $17 / 22(77.2)$ & $26 / 30(86.7)$ & $0.380 * * *$ \\
\hline \multicolumn{4}{|l|}{ of antifungals } \\
\hline Ophthalmological & $5 / 22(22.7)$ & $21 / 30(70.0)$ & $<0.001 * * *$ \\
\hline \multicolumn{4}{|l|}{ examinations } \\
\hline Repeat blood & $10 / 22(50.0)$ & $22 / 30(73.3)$ & $0.040 * * *$ \\
\hline \multicolumn{4}{|l|}{ cultures after the } \\
\hline \multicolumn{4}{|l|}{ start of therapy } \\
\hline Assessment of & $21 / 22(92.9)$ & $26 / 30(86.6)$ & $0.290^{* * *}$ \\
\hline
\end{tabular}


clinical

effectiveness on the

third to fifth day

14 days of therapy $\quad 7 / 22(31.8) \quad 16 / 30(53.3) \quad 0.120^{* * * *}$

after blood culture

is confirmed to be

negative

Key 7 elements ** $\quad 3 / 22(13.6) \quad 15 / 30(50.0) \quad 0.006 * * *$

25 Data are presented as No. of patients (\%)

$26{ }^{* * * *}$ Patients with CV catheter placement

$27{ }^{* * * *}$ Patients without CV catheter placement were 6 elements

$28 * * *$ Chi-square test 